IOSR Journal of Pharmacy

e-ISSN: 2250-3013, p-ISSN: 2319-4219, www.iosrphr.org

Volume 2 Issue 6 || || Nov-Dec. 2012 || || PP.21-32

\title{
Preparation and characterization of fibrin-chitosan composite and its in vivo studies
}

\author{
${ }^{1}$ Pandima Devi M, ${ }^{2}$ T.P.Sastry, Meignanalakshmi S* \\ ${ }^{1}$ Research Scholar, Bharath University, Selaiyur, Chennai \\ ${ }^{1 *}$ Department of Biotechnology, SRM University, Kattankulathur \\ ${ }^{2}$ Head, Bioproducts Lab, Central Leather Research Institute, Chennai
}

\begin{abstract}
Biomaterials are used in regenerative medicine, implantable materials, controlled release carriers or scaffolds for tissue engineering. Biological dressings like fibrin glue, gelatin sheets, chitosan films, collagen is popular for quicker wound healing. These polymers when used in combination such as fibringelatin, fibrin-chitosan has shown better results, than when used alone. Thus, the present research work is concentrated on the preparation and evaluation of composite films made with chitosan-fibrin to ascertain the applicability of the prepared combination for wound management. Temporary biological wound dressings were prepared, in film form, using physiologically clotted fibrin and chitosan (FC) employing PVP as porogen. The films were characterized for their percentage equilibrium water content (\% EWC), water vapour transmission rate (WVTR), bacterial penetration, surface morphology and in vivo biodegradation using rats as animal models. The film prepared using fibrin $10 \mathrm{ml}$ (40\% solids) and chitosan solution $5 \mathrm{ml}$ (4\% in $0.3 N$ acetic acid) has exhibited better results. The FC films have shown excellent inhibition for bacterial penetration. The micrographs taken for wet membrane have shown swollen strands of fibrin expanding throughout the surface indicating the hydrogel nature of the film. In vivo degradation studies have revealed that the FC is completely biodegradable in 30 days without any adverse foreign body reaction indicating its biocompatibility.
\end{abstract}

Keywords - Chitosan, fibrin, equilibrium water content (\% EWC), water vapour transmission rate (WVTR), bacterial penetration, surface morphology, in vivo biodegradation

Biomaterials

\section{INTRODUCTION}

The use of natural polymers, or biopolymers, as structural materials is not new. Nature itself has always used, for instance, cellulose to provide the structure of higher plants, chitin as the exoskeleton of several molluscs, keratin for thermoinsulation in hair, collagen for mechanical support in connective tissues, and silk in spiderwebs. At present, the socio-economic situation of the modern world has raised the interest in these materials. Oil embargos and high prices, associated with the concerns of oil long-term availability, are favorable to the replacement of oil-derived polymers by polymers derived from renewable resources. Environmental concerns are also playing an increasingly important role, contributing to the growing interest in natural polymers due to their biodegradability, low toxicity and low disposal costs. The usually low manufacture costs of biopolymers, related to their large agricultural availability and renewability, are additional advantages. Furthermore, their versatility of chemical structures and their well-known chemistry allow the development of advanced functionalized materials that can match several varied requirements. In addition, the rapid advancement in understanding of fundamental biosynthetic pathways through genetic manipulations will provide tailoring of biopolymer structure and function, thus creating new opportunities for these materials (Kwunchit,1997), Tokura,1996), and (Xu,1996).

In the biomedical field, the degradation of natural polymers into physiological metabolites makes them excellent candidates for a wide range of applications, such as drug delivery. Polysaccharides, in particular, have some excellent properties which make them the polymer group with the longest and widest medical applications experience: Georgiev ,2000, Bulter 1996, and Gong, 2000 non-toxicity (monomer residues are not hazardous to health), water solubility or high swelling ability by simple chemical modification, stability to $\mathrm{pH}$ variations, and a broad variety of chemical structures. This versatility makes these materials able to overcome some disadvantages like low mechanical, temperature and chemical stability, and proneness to microbial and enzymatic degradation, which, in some cases, can be used as an advantage. 


\section{Wound dressing Materials}

From the ancient times, for effective healing of a wound, a suitable material had to be used to cover the wound in order to prevent any infection. Historically, honey pastes, plant fibers, and animal fats were used as wound dressing materials (G. Majno, 1975). The first event to follow tissue injury is hemorrhage from injured vessels. The subsequent coagulation process produces a fibrin network that fills the wound space, attaches to the wound surfaces and in some instances acts as biological glue keeping wound edges together. In the case of tissue defect, the wound edges cannot be brought together and the defect is then filled by the coagulum forming a scab which occludes the wound. The inflammatory exudate accumulating in traumatized tissues is rich in fibrinogen, which is converted to fibrin. Fibrin is thus an integrated part of every reparative process. Application of exogenous fibrin to wound surfaces may assist hemostasis and be positive for healing by avoiding hematomas. Degradation products of fibrin do activate macrophages, which are an important part of the wound cell population by contributing growth factors. It may be of great interest that the chemical structure of fibrin has some similarities to growth factors. Indirect evidence is thus strong that fibrin plays an important role in wound healing process (Schalag, 1986). The possible role of fibrin as a basis for ingrowing fibroblasts has often been pointed out, but the problems involved are poorly understood. (Peacock et al 1976) There is however a close association between migrating and proliferating fibroblasts and the fibrin network. A special feature of fibrin both formed naturally (or) therapeutically, are that it is degraded and remodelled by cell associated enzymatic activity during cell migration and wound healing (Ye et al 2000). Nowadays, with new biopolymers and fabrication techniques, a wound dressing material is expected to have extraordinary properties which enhance the healing process of a wound. For an effective design of a functional wound bandage, characteristics of the wound type, wound healing time, physical, mechanical, and chemical properties of the bandage must be taken into consideration. Ultimately, the main purpose is to achieve the highest rate of healing and the best aesthetic repair of the wound (Thomas et al. 1990). To perform these varied functions in wound dressings, the biocomposites should meet the following requirements: (1) biocompatibility with the tissues, (2) biodegradability at the ideal rate corresponding to the rate of new tissue formation, (3) nontoxicity and nonimmunogenicity, (4) optimal mechanical property, and (5) adequate porosity and morphology for transporting of cells, gases, metabolites, nutrients and signal molecules both within the biocomposites and between the biomaterial and the local environment.

It was reviewed that many of the dressings and novel polymers used for the delivery of drugs to acute, chronic and other types of wound. These include hydrocolloids, alginates, hydrogels, polyurethane, collagen, chitosan, pectin and hyaluronic acid. Pharmacological agents such as antibiotics, vitamins, minerals, growth factors and other wound healing accelerators that take active part in the healing process were discussed. Direct delivery of these agents to the wound site is desirable, particularly when systemic delivery could cause organ damage due to toxicological concerns associated with the preferred agents. This review concerns the requirement for formulations with improved properties for effective and accurate delivery of the required therapeutic agents. General formulation approaches towards achieving optimum physical properties and controlled delivery characteristics for an active wound healing dosage form are also considered briefly (Boateng et al 2008). It was studied that the chitosan based dressings are used for hemorrhage control in the wound management. There were two reported dressing failures that occurred with blind application of bandages up into large cavitational injuries. Dressings were reported to be most useful on areas where tourniquets could not be applied to control bleeding. The dressings were reported to be most difficult to use in extremity injuries where they could not be placed easily onto or into the wounds. No complications or adverse events were reported (Wedmore, 2006).

\section{Natural Polymers}

Recently, attention has been focused on employing natural polymers such as collagen, (Cuadrillero, 2004, Sripriya et al 2004) gelatin, (Changez et al 2004, Gyayamzadeh et al 2004) chitosan, (Jayasree et al 1995, Minami et al 1992), hyaluronic acid (Segura et al 2005, Hahn et al 2004) and fibrin (Bence et al 1994, Sathian et al 2003, Rothwellet al 2002) etc., to prepare biodegradable wound dressing materials. Among the abovementioned natural polymers, fibrin in its natural state (physiologically clotted) is abundantly available as a byproduct in meat industry. The therapeutically utilization of this fibrin is not yet properly achieved. This product is nothing but the blood clot formed after any injury caused to the body. Fibrin behaves as a provisional matrix and attracts platelets and monocytes to itself during healing process and plays a crucial role in wound healing (Martin, 1997).

One of the methods adopted to enhance the biocompatibility of artificial materials, especially synthetic wound dressing materials is the application of a thin, crosslinked, fibrin surface coating to the material. It has been proved that the stability of the material increases if the fibrin is crosslinked (Rubens et al 1995). Hydrogels based on physiologically clotted fibrin were prepared by us using various monomers, characterized and used them as wound dressing materials on the experimental wounds of rats. The results have shown that these hydrogels induced the proliferation of fibroblasts and thereby enhanced the wound healing process. In these 
studies gelatin was used as a reinforcement material, as fibrin alone could not form a continuous film (Noorjahan et al 2004).

In the present study chitosan is used as the reinforcement material in place of gelatin as it exhibits bacteriostatic and fungistatic activities (Tomihata et al 1997, Muzzarelli et al 1990) besides hemostatic and tissue regeneration activities (William et al 1985). Chitosan has been examined to be used in a wide variety of biomedical applications, such as drug delivery carriers, surgical thread, bone-healing materials, especially wound dressing (Biagini et al 1991, Berthod et al 1993, Abhay 1998, Su et al 1997). Chitosan is a semi-crystal polymer and its membrane has lower gas permeability in original state (Muzzarelli et al 1974). However, the gas exchange through a wound dressing is important, because a high $\mathrm{CO}_{2}$ pressure increases the acidity and slows down the healing process, and in addition a low oxygen concentration decreases the regeneration of tissue cell or makes possible the proliferation of anaerobic bacteria (Mi et al 2001). Hence polyvinyl pyrrolidone (PVP)-a porogen, was incorporated into the fibrin-chitosan composite in order to increase the porosity of the material.

It was studied that the composites, in film form, containing physiologically clotted fibrin, chitosan and gelatin were prepared and crosslinked with glutaraldehyde. A composite film prepared from the mixture of fibrin and chitosan showed superior characteristics comparing with fibrin and chitosan films, respectively. A film prepared from fibrin was too fragile to handle without any additive. The films were characterized for their IR spectroscopy, water absorption capacity (WAC) at different $\mathrm{pH}$ conditions, mechanical properties and scanning electron microscopy (SEM). Fibrin and gelatin films gave higher WAC values in all the pH ranges (2, 7 and 10) studied. However, with the addition of chitosan, the WAC values of the composite decreased. This was attributed to the crosslinking of glutaraldehyde with the hydrophilic groups available on chitosan and gelatin/fibrin. The amount of individual constituents, which gave maximum tensile strength to the FCG composite, was optimized. SEM pictures of the FCG have exhibited the fibrous and porous nature of the composite (Natarajan et al 2005).

It was reported that to create a scaffold that is suitable for the construction of tissue-engineered skin, a novel asymmetric porous scaffold with different pore sizes on either side was prepared by combining a collagen-chitosan porous membrane with fibrin glue. Tissue-engineered skin was fabricated using this asymmetric scaffold, fibroblasts, and a human keratinocyte line. Epidermal cells could be seen growing easily and achieved confluence on the fibrin glue on the upper surface of the scaffold. Scanning electron microscopy showed typical shuttle-like fibroblasts adhering to the wall of the scaffold and fluorescence microscopy showed them growing in the dermal layer of the scaffold. The constructed composite skin substitute had a histological structure similar to that of normal skin tissue after three weeks of culture. The results of the study suggest that the asymmetric scaffold is a promising biologically functional material for skin tissue engineering, with prospects for clinical applications (Chun-Mao Han, 2010).

The three-dimensional collagen-chitosan scaffolds were fabricated with type I collagen and chitosan through freeze drying and glutaraldehyde cross-linking. Dermal fibroblasts were isolated from neonatal Sprague-Dawley rat skin by dispase II/collagenase I digestion. The fibroblasts were then seeded into the scaffolds to construct tissue-engineered dermis. The microstructure of the scaffolds as well as the fibroblasts' proliferation, cytokine secretion and cell cycle were investigated. Flow cytometry analysis indicated that cells in the scaffolds proliferated steadily. IL-6 concentration measurement by the ELISA test suggested that the scaffolds could promote secretion of the fibroblasts' cytokine. These results show that the fibroblasts and the scaffolds interact well with each other, and the fibroblasts have better proliferation ability and biological activity in the scaffolds than in monolayer culture. The scaffolds are a promising candidate for tissue repair and regeneration with enhanced biostability and good cytocompatibility (Sun LP, 2009).

The fast development of tissue engineering and regenerative medicine drives the old biomaterials, for example, fibrin glue, to find new applications in these areas. It was reported that the developing a commercially available hydrogel for cell entrapment and delivery and optimized the fabrication and gelation conditions of fibrin gel. Fibrinogen was isolated from human plasma by a freeze-thaw circle. Gelation of the fibrinogen was accomplished by mixing with thrombin. Absorbance of the fibrinogen/thrombin mixture at $550 \mathrm{~nm}$ as a function of reaction time was monitored by UV-VIS spectroscopy. It was found that the clotting time is significantly influenced by the thrombin concentration and the temperature, while less influenced by the fibrinogen concentration. After freeze-drying, the fibrin gel was characterized by scanning electron microscopy (SEM), revealing fibrous microstructure. Thermal gravimetric analysis found that the degradation temperature of the crosslinked fibrin gel starts from $288^{\circ} \mathrm{C}$, which is about $30^{\circ} \mathrm{C}$ higher than that of the fibrinogen. The hydrogel has an initial water-uptake ratio of $\sim 50$, decreased to 30-40 after incubation in water for $11 \mathrm{~h}$ depending on the thrombin concentration. The fibrin gels lost their weights in PBS very rapidly, while slowly in DMEM/fetal bovine serum and DMEM. In vitro cell culture found that human fibroblasts could normally proliferate in the fibrin gel with spreading morphology. In conclusion, the fibrin gel containing higher concentration of fibrinogen $\left(20 \mathrm{mg} \mathrm{ml}^{-1}\right)$ and thrombin $\left(5 \mathrm{U} \mathrm{ml}^{-1}\right)$ has suitable gelation time and handling properties, and thus is applicable as a delivery vehicle for cells such as fibroblasts (Haiguang Zhao, 2008). 
The recent efforts focused on the chemical and biological modification of chitosan in order to increase its solubility in aqueous solutions and absorbability in the in vivo system, thus for a better use of chitosan. Chitosan modification and its pharmaceutical/biomedical applications are summarized in a review based on the achievements as well as the domestic and overseas developments: (1) enzymatic preparation of low molecular weight chitosan/chitooligosaccharides with their hypocholesterolemic and immuno-modulating effects; (2) the effects of chitin, chitosan and their derivatives on blood hemostasis; and (3) synthesis of a non-toxic ion ligand-D-Glucosaminic acid from Oxidation of D-Glucosamine for cancer and diabetes therapy (Jiali Zhang, 2010).

It was reported that the chitosan dressing by incorporating a procoagulant (polyphosphate) and an antimicrobial agent (silver) improve its hemostatic and antimicrobial properties. The study demonstrated for the first time, the application of polyphosphate as a hemostatic adjuvant, and developed a new chitosan-based composite with potent hemostatic and antimicrobial properties (Ong et al, 2008).

It was reported that the polymers are applicable as a wound dressing to protect the wound site against unwanted external effects, inhibit wound contraction, and, if possible, stimulate the healing process. Micro- and nanoparticulates are currently being evaluated as a potential drug delivery in clinical applications. Growth factors also play a vital role in wound healing. Polymers used in wound healing act as sustained release vehicles for growth factors. Controlled release of growth factors from microspheres has provided a higher degree of healing in the wound areas. (Degim, 2008).

It was investigated that the antimicrobial mode of action of chitosan using a combination of approaches, including in vitro assays, killing kinetics, cellular leakage measurements, membrane potential estimations, and electron microscopy, in addition to transcriptional response analysis. Chitosan, whose antimicrobial activity was influenced by several factors, exhibited a dose-dependent growth-inhibitory effect (Dina Raafat, 2008).

It was observed that the use of the chitosan has low oral toxicity and local tolerance potential supporting use in non-parenteral formulations. Prior human oral exposure has occurred through use of chitosan dietary supplements and food additive, medical device and cosmetic applications. Although systemic exposure to parent chitosan may be limited (due to digestion in the gastrointestinal tract) to any that is absorbed will likely undergo enzyme degradation to naturally occurring glucosamine, and $N$-acetylglucosamine, its copolymers, which are excreted or used in the amino sugar pool. Chitosan has local biological activity in the form of haemostatic action and, together with its ability to activate macrophages and cause cytokine stimulation (which has resulted in interest in medical device and wound healing applications), may result in a more careful assessment of its safety as a parenteral excipient (Paul Baldrick, 2010).

In the present study a fibrin-chitosan (FC) wound dressing material was prepared. Thus prepared FC wound dressing was characterized for its percentage Equilibrium Water Content (\% EWC), Water Vapour Transmission Rate (WVTR), bacterial penetration, surface morphology and chemical composition. One of the important prerequisites for the clinical usefulness of a wound dressing is its good biocompatibility. In vivo biocompatibility and biodegradation evaluations are commonly based on subcutaneous implantation studies (Anderson 1994). Hence the biocompatibility and biodegradation evaluations of FC wound dressing were carried out by subcutaneously implanting the same in rats. Histological sections and Scanning Electron Micrographs of the FC composite after implantation were analyzed.

\subsection{Materials and methods}

\section{EXPERIMENTAL}

Crude fibrin was collected from a Municipal corporation slaughter house (Chennai, India) and chitosan was prepared from prawn shells. All other reagents used were of analytical grade.

\subsection{Purification of crude fibrin}

Physiologically clotted fibrin was purified as described elsewhere (Ravindra Babu et al 1989). Briefly, the crude fibrin that was separated from fresh blood by churning was collected from the slaughter-house and washed thoroughly under running water to remove the blood clots. Later the fibrin was treated with $0.5 \mathrm{M}$ sodium acetate solution and $20 \%$ hydrogen peroxide solution. The fibrin was then washed thoroughly under running water and ground to a paste in a mixer. This paste contained $40 \%$ solids.

\subsection{Preparation of chitosan from prawn shells}

Chitosan was prepared by the modification of earlier method (Mochizuki et al 1989). The prawn shells were cleaned thoroughly with water to remove sand and impurities. Then the shells were treated with $5 \%$ sodium hydroxide solution for about $2 \mathrm{~h}$ for deproteinization. The product was washed well with water and treated with hydrochloric acid solution $(2 \mathrm{~N})$ to remove calcium carbonate. The resultant product was chitin. 
The pure chitin thus obtained was treated with $40 \%$ Sodium hydroxide and heated in an oil bath maintained at about $180^{\circ} \mathrm{C}$ for $2 \mathrm{~h}$. The contents were routinely checked for their solubility in hydrochloric acid $(0.1 \mathrm{~N})$ solution. Once the contents were dissolved in hydrochloric acid, the same was removed from the bath, washed thoroughly with water and dried well. The resultant product was chitosan.

\subsection{Preparation of Fibrin-Chitosan composite (FC)}

Fibrin after purification was used to prepare Fibrin-Chitosan composite (FC). The amount of fibrin taken was varied between $5-25 \mathrm{ml}(40 \%)$, whereas the amount of chitosan $5 \mathrm{ml}(4 \%$ chitosan solution in $0.3 \mathrm{~N}$ acetic acid) and that of polyvinyl pyrolidone (PVP) $5 \mathrm{ml}$ (5\% in water) were kept constant. To the fibrin paste, chitosan solution and PVP solution were added and the contents were mixed and cast into a film using polythene tray measuring $5 \times 10 \mathrm{~cm}^{2}$. After drying, the film was placed in $0.2 \%$ sodium carbonate solution at room temperature for 48 hour on a roller mixer and rinsed three times in phosphate buffer saline in order to bring the $\mathrm{pH}$ of the film to 7.4. Finally, the membrane was kept in water with the bath temperature $80^{\circ} \mathrm{C}$ for more than $8 \mathrm{~h}$ to dissolve the PVP, the porogen and to generate porous membrane. By the heat treatment selective dissolution of PVP is accelerated and the remaining matrix, which contains fibrin and chitosan, remains intact, as they are insoluble in water. The films were dried at $60^{\circ} \mathrm{C}$ under vacuum, sterilized by $\gamma$ irradiation at $2 \mathrm{M}$ rads and sealed under sterile atmosphere.

\subsection{Fourier Transform Infra Red Spectroscopy}

FTIR (Fourier-transform IR) studies on the samples were carried out using Nicolet Impact 400 FTIR spectroscopy. The Nicolet Impact 400 FT-IR is a very high end optical bench with a range of 7400-375 $\mathrm{cm}^{-1}$. The Frequency Range is $400-5000 \mathrm{~cm}^{-1}$, the Spectral Resolution is $0.125 \mathrm{~cm}-1$ and the Beam splitters is $\mathrm{KBr}$ $\left(375-7000 \mathrm{~cm}^{-1}\right)$.

\subsection{Equilibrium water content}

The water absorbing capacity of FC sheet is quantitatively represented by the equilibrium water content (EWC) which is represented as the ratio of the weight of water in the hydrogel to the weight of the hydrogel at equilibrium hydration. The EWC was measured by the weight difference between the swollen hydrogel and the dehydrated polymer as described earlier (Corkhill et al 1994). This is expressed as

$$
\text { EWC }(\%)=\quad \times 100 \quad \begin{aligned}
& \text { Weight of water in hydrated gel } \\
& \text { Total weight of hydrated gel }
\end{aligned}
$$

\subsection{Measurement of Water Vapor Transmission Rate}

The water vapor transmission rate (WVTR) was measured according to monograph of the European Pharmacopeia (Gennadios et al 1994). It consisted of measuring the weight loss of a bottle, which contain $25 \mathrm{ml}$ water. The bottle had a mouth with a diameter of $40 \mathrm{~mm}$ was then put at the bottle mouth as a cap, and placed in an oven at $35^{\circ} \mathrm{C}$ for 24 hour. The water vapor transmission rate was calculated as follows

$$
\text { WVTR }=\frac{\left(\mathrm{W}_{\mathrm{i}}-\mathrm{W}_{\mathrm{t}}\right) \times 10^{6}}{\mathrm{~A} \times 24} \mathrm{~g} / \mathrm{m}^{2} \mathrm{~h}
$$

Where WVTR is expressed in $\mathrm{g} / \mathrm{m}^{2} \mathrm{~h}, \mathrm{~A}$ is the area of bottle mouth $\left(\mathrm{mm}^{2}\right), \mathrm{W}_{\mathrm{i}}$ and $\mathrm{W}_{\mathrm{t}}$ are the weight of bottle before and after placed in oven, respectively.

\subsection{Bacterial penetration test}

Sterilized samples with diameter of $1.5 \mathrm{~cm}$ were cut from FC wound dressings and placed on agar plates. Subsequently, the sample was inoculated with a suspension $(0.2 \mathrm{ml})$ of an overnight culture of Pseudomonas aeruginosa $\left(10^{8}-10^{9}\right.$ bacteria / ml $)$ on its center. After one week of incubation at $25^{\circ} \mathrm{C}$ the films were checked for bacterial penetration by using specific reagents, which promote the production of fluorescent green pigment (Pyoverdine) and blue-green pigment (Pyocyanine) (Mi et al 2001).

\subsection{Surface morphological studies}

i) Light microscopy

The xerogels and hydrogels of $\mathrm{F}_{10} \mathrm{C}$ film were viewed and micrographs were taken at 10x magnification using Nikon microscope, type 115 .

\section{ii) Scanning Electron Microscopy (SEM)}

The dried film (initial) and the tissues at the implant site (after 15 and 30 Post Implantation Days) were gold coated using a sputter coater (fisons sputter) under the following conditions. 0.1 Torr pressure, $200 \mathrm{~mA}$ 
current and 70s coating time. Surface structure was visualized by scanning electron microscope (SEM modelLEICA stereo scan 440) using a $15 \mathrm{KV}$ accelerating volt.

\subsection{AFM (Atomic Force Microscopy)}

The particle size distribution of the Chitosan fibrin composite was analysed using AFM. The studies in contact mode were carried out using an atomic force microscope (Agilent): model, Pico SPM; controller model, Pico scan, serial no. 305-0002532; microscope model, Pico SPM, serial no. 300-0603357; software version, Pico scan version 5.4. The cantilever was from Nova Scan Technologies: material, silicon nitride; scan rate, 2.4 $\mu \mathrm{m} / \mathrm{s}$; scanned area, $5 \times 5,2 \times 2$ and $1 \times 1 \mu \mathrm{m} 2$; data points, 256 per line.

\subsection{In vivo degradation and biocompatibility}

The FC films of dimensions $1 \mathrm{~cm} \times 1 \mathrm{~cm} \times 0.06 \mathrm{~mm}$ were implanted on the backs of Albino rats $(250 \pm$ $10 \mathrm{~g}$ ). Animals were anesthetized by intraperitoneal injection with a mixture of Ketamin HCL ( $85 \mathrm{mg} / \mathrm{kg}$ body weight) and Xylazine (12 $\mathrm{mg} / \mathrm{kg}$ body weight). The rats were shaved and betadine solution (10\%) was applied. A $10 \mathrm{mg} / \mathrm{kg}$ dose of tetracycline was given at the time of surgery. Two incisions (approx. $1.5 \mathrm{~cm}$ long) $1.5 \mathrm{~cm}$ apart was made laterally about the mid portion of the back as represented in the schematic diagram (Fig.3.1). A pocket was formed subcutaneously around each incision and the film was inserted. Each incision was closed by intermittent sutures with surgical nylon thread and a No. 11 surgical blade, $0.5 \mathrm{~cm}$ apart. Implanted films were explanted at 5, 10,15 and 30 days for analysis. One of the two explanted films from each rat were utilized for doing histology while the other was utilized to take SEM micrographs. For histological studies, tissues surrounding the implanted films were placed in $10 \%$ formalin for $48 \mathrm{~h}$, dehydrated serially, paraffin embedded, sectioned and stained with hematoxylin and eosin. Samples were examined with light microscopy by an expert pathologist to monitor the tissue reactions. For SEM studies tissues after explanting were washed with PBS and then dried by serial dehydration using ethanol. Three samples per time point were used for the in vivo biodegradation study.

\section{RESULTS AND DISCUSSION}

\section{1 Percentage Equilibrium Water Content (\%EWC)}

Exudate drainage ability of FC films was examined by carrying out the water absorption of the films in Phosphate Buffered Saline solution (PBS) of pH-7.4. Table 1 shows the \% EWC of the FC films. The higher water content exhibited by the FC films between $54-66.6 \%$ would be attributed to the presence of both macro and micro pores formed due to the presence of fibrin and selective leaching of PVP from FC films respectively.

Tabl.3.1 Results of water absorption, transmission and bacterial permeability properties of FC films

\begin{tabular}{|l|l|l|l|l|l|l|l|}
\hline $\begin{array}{l}\text { Sample } \\
\text { Code }\end{array}$ & $\begin{array}{l}\text { Fibrin } \\
(\mathbf{m l})\end{array}$ & $\begin{array}{l}\text { Chitosan } \\
(\mathbf{m l})\end{array}$ & $\begin{array}{l}\text { PVP } \\
(\mathbf{m l})\end{array}$ & $\begin{array}{l}\text { Thickness } \\
(\mathbf{m m})\end{array}$ & $\begin{array}{l}\text { \% } \\
\text { EWC }\end{array}$ & $\begin{array}{l}\text { WVTR } \\
\mathbf{g} / \mathbf{m}^{\mathbf{2}} \mathbf{h}\end{array}$ & $\begin{array}{l}\text { Bacterial } \\
\text { Permeability }\end{array}$ \\
\hline $\mathrm{F}_{5} \mathrm{C}$ & 5 & 5 & 5 & 0.04 & 54.5 & 2118.2 & Impermeable \\
\hline $\mathrm{F}_{10} \mathrm{C}$ & 10 & 5 & 5 & 0.06 & 66.6 & 2383.2 & Impermeable \\
\hline $\mathrm{F}_{15} \mathrm{C}$ & 15 & 5 & 5 & 0.12 & 61.9 & 2339.8 & Impermeable \\
\hline $\mathrm{F}_{20} \mathrm{C}$ & 20 & 5 & 5 & 0.17 & 60.9 & 2287.8 & Impermeable \\
\hline $\mathrm{F}_{25} \mathrm{C}$ & 25 & 5 & 5 & 0.25 & 56.2 & 2149.2 & Impermeable \\
\hline
\end{tabular}

Fibrin solution - $40 \%$ in water, chitosan solution - $4 \%$ in $0.3 \mathrm{~N}$ acetic acid, PVP solution - $5 \%$ in water

\subsection{Water vapor transmission rate (WVTR)}

Table 3.1 represents the WVTR exhibited by FC films. With the increase in the fibrin content of the film, the WVTR initially increases and then decreases. The film $\mathrm{F}_{10} \mathrm{C}$ exhibited the highest WVTR and the value being $2383.2 \mathrm{~g} / \mathrm{m}^{2} \mathrm{~h}$. The permeation of water vapour through a hydrogel consists of two steps adsorption and diffusion. Due to the presence of the pores the water first gets adsorbed and depending on the thickness, diffusion takes place. The WVTR is higher for thin porous films. As $\mathrm{F}_{10} \mathrm{C}$ has exhibited better \%EWC and WVTR the same was selected for further characterization studies.

\subsection{Bacterial penetration}

The in vitro bacterial penetration tests have shown that all FC wound dressings have the ability to prevent wound from penetration of bacteria. No colour developed on the other side of the membrane after 7 days, suggesting that Pseudomonas aeruginosa does not cross the membrane (Table3.1). This impermeability for bacterial penetration would be attributed to the inherent antibacterial property of chitosan. Hence the results indicated that the FC films could exhibit excellent inhibition of bacteria penetration. 


\subsection{Surface morphology analysis}

Figure 3.1a represents the micrographs of $\mathrm{F}_{10} \mathrm{C}$ xerogel and Fig. 3.1b represents that of hydrogel. Interconnected macropores filled with water could be seen. The swollen strands of fibrin expanding through out the surface could also be visualized. Fig. 3.1c represents the scanning electron micrograph of dried $\mathrm{F}_{10} \mathrm{C}$ film (initial). It is well known that fibrin is fibrous in nature with a crosslinked system. The macropores seen on the surface of the film is due to the presence of fibrin matrix.
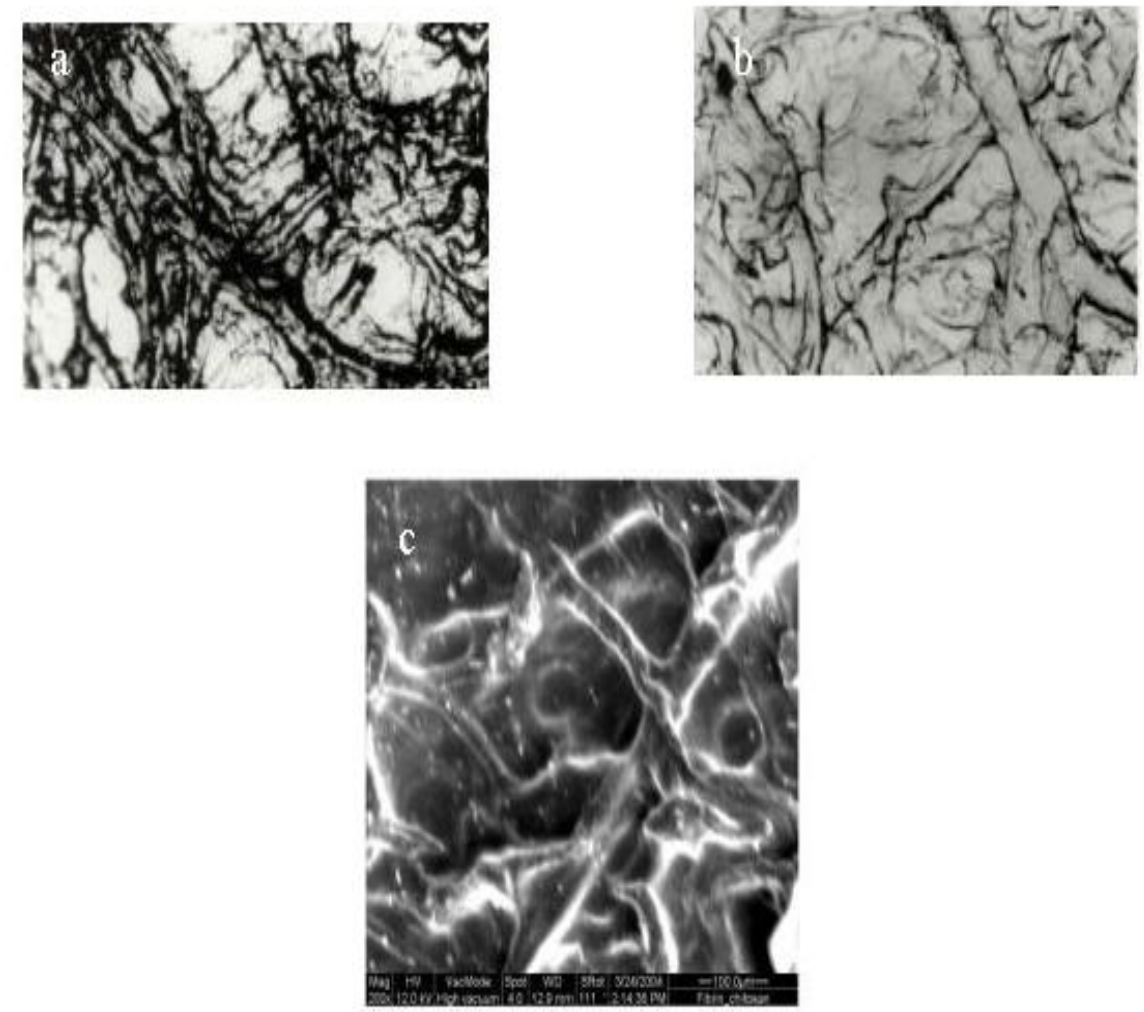

Fig. 3.1Surface morphology of $F_{10} C$ film a) Photomicrograph of xerogel b) Photomicrograph of hydrogel c) Scanning electron micrograph (initial)

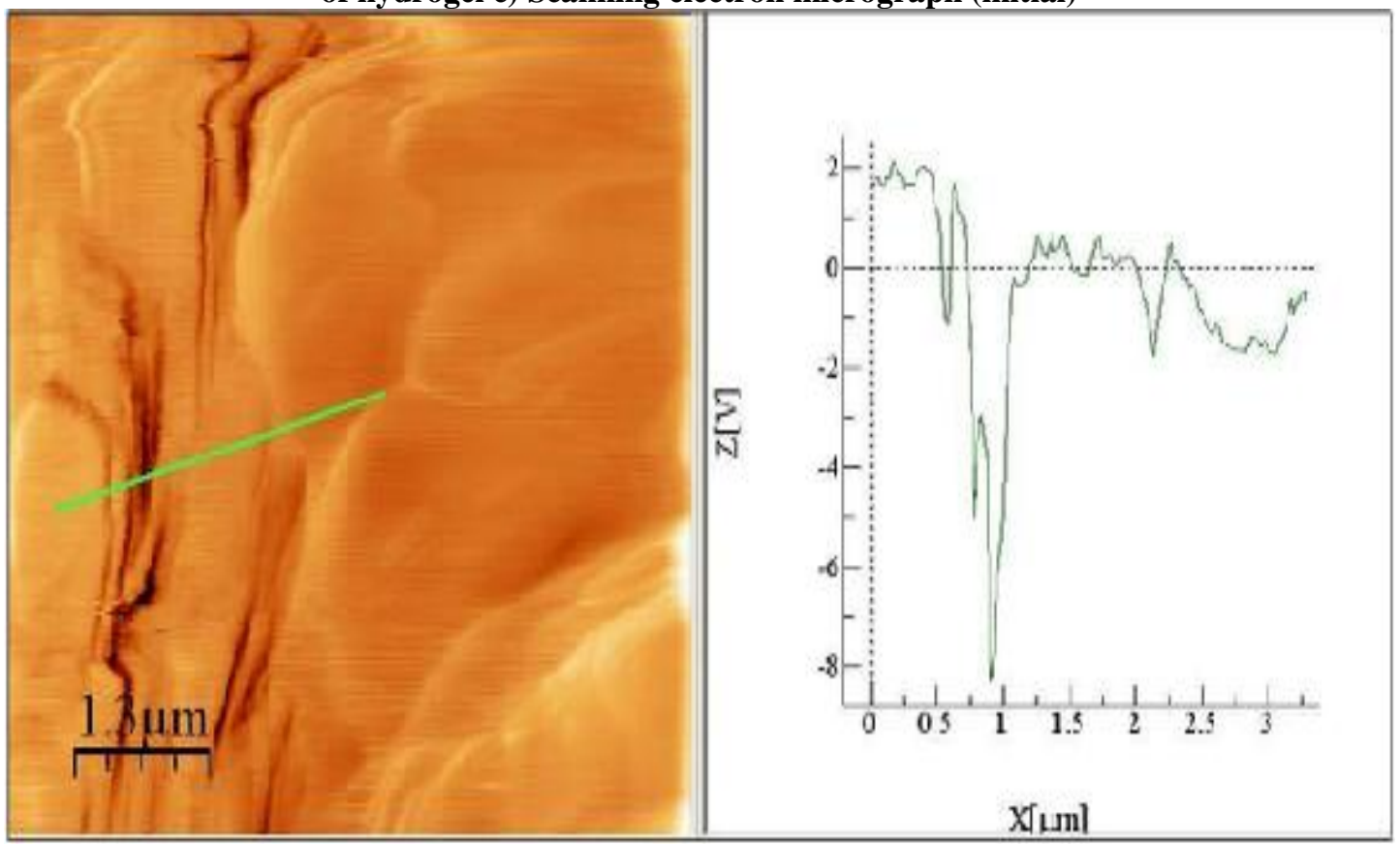


Fig. 3.2 AFM Analyses: (10ml Fibrin and 5ml Chitosan)

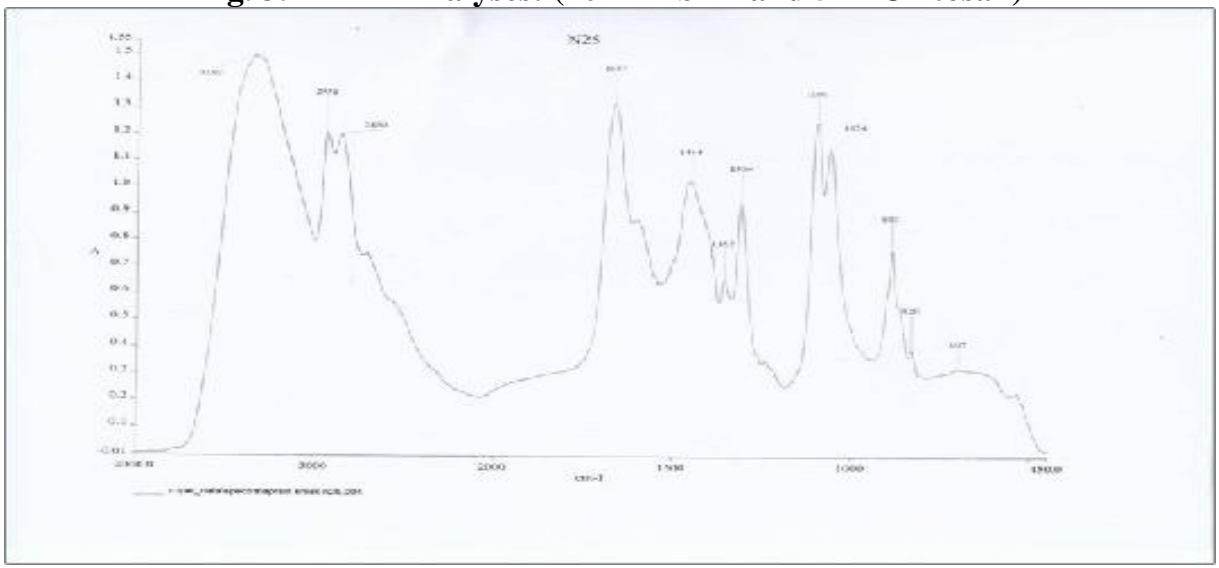

Fig. 3.3 FTIR (10ml Fibrin and 5ml Chitosan)
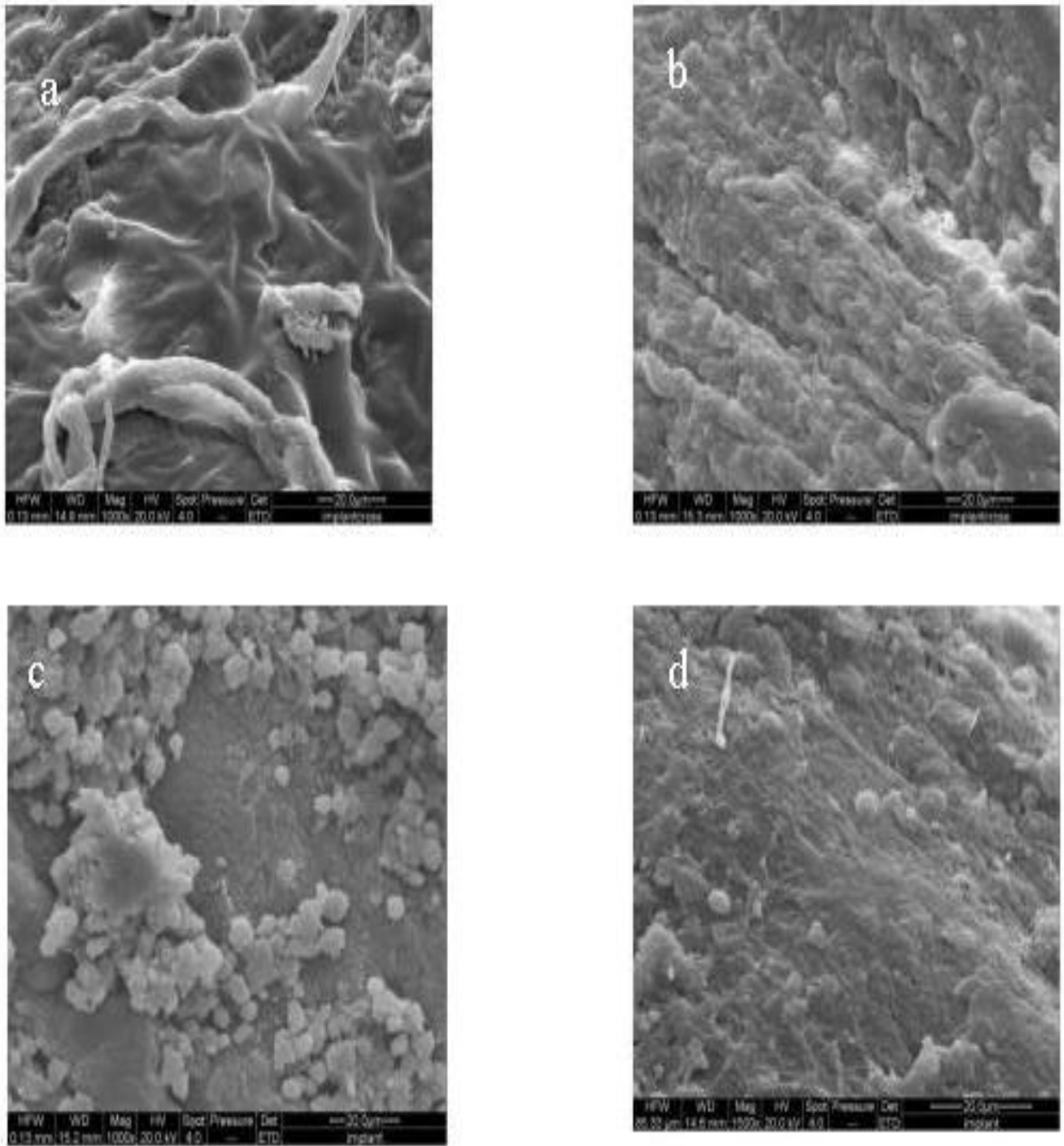

Fig. 3.4 scanning electron micrographs of in vivo degraded $F_{10} C$ film a) and b) represent $15^{\text {th }}$ PID whereas c) and d) represent $30^{\text {th }}$ PID 

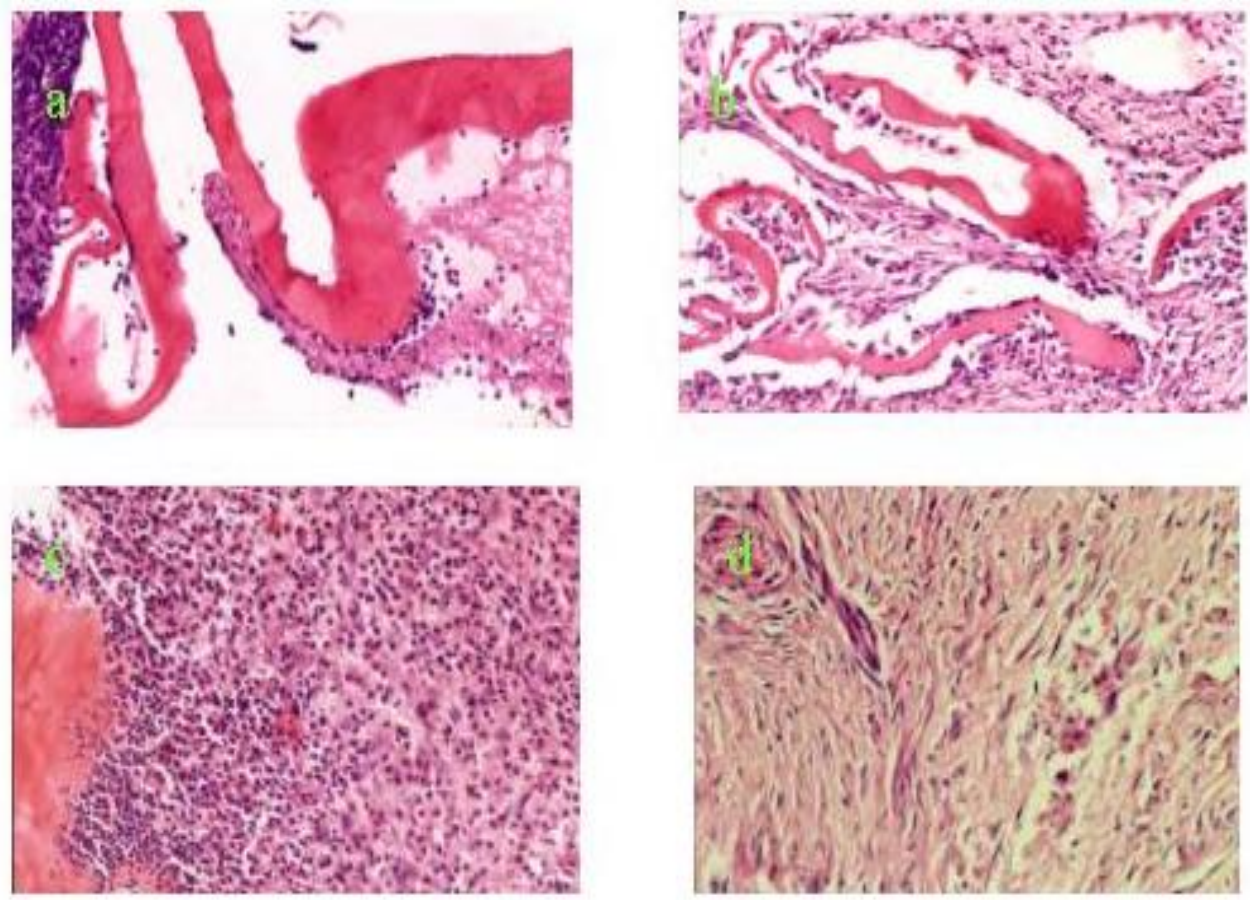

Fig. 3.5 Photomicrographs of in vivo degraded $\mathrm{F}_{10} \mathrm{C}$ film taken on a) $5^{\text {th }}$, b) $10^{\text {th }}$, c) $15^{\text {th }}$ and d) $30^{\text {th }}$ post implantation days

\section{DISCUSSION}

It was observed from the fig: 3.3, that peaks 3368, 2951,1649,1392,1302 $\mathrm{cm}^{-1}$ which represents the $\mathrm{O}-\mathrm{H}, \mathrm{O}-\mathrm{H}, \mathrm{C}=\mathrm{O}$ and $-\mathrm{NO}_{2}$ groups. The aminoacid groups has a characteristic adsorption band in the region of $3400-3500, \mathrm{~cm}^{-1}$ which is masked by the broad adsorption band from the O-H group. At 1649, it shows that the chitosan has highest peak, which means it is partially deacytelated product. The evidence of FTIR measurements also points to the formation of new resonance that cannot be accounted for in a simple mixture.

\section{(Naomi Edelman, 2004)}

The FTIR spectrum of fibrin chitosan showed in the fig.3.3 the characteristic peaks of both chitosan and fibrin. The amide adsorption bands of fibrin are seen at $1657 \mathrm{~cm}^{-1}$ (amide-I) and $1550 \mathrm{~cm}^{-1}($ amide II). The skeletal vibration involving C-O of the chitosan is seen around $1070 \mathrm{~cm}^{-1}$. A broad band between $3200 \mathrm{~cm}^{-1}-3400 \mathrm{~cm}^{-1}$ represent stretching of $-\mathrm{OH}$ bonds present in chitosan and fibrin. As these bands are overlapped, it is seen as broad band. This spectrum clearly shows presence of both fibrin and chitosan.

The FTIR spectrum of chitosan and fibrin contain all the characteristics absorption peaks of chitosan and fibrin. As there are no shifts of peaks of any groups of any group in the composite spectrum. It is confirmed that chitosan and fibrin composite is only a mixture and no chemical reaction has taken place between the individual components.

\subsubsection{AFM Morphology}

To obtain information on the micro and nano scale surface structure, tappin mode AFM imaging was employed to determine morphology and roughness information of the composite film. The topographical nature of the biofilm was observed by using the AFM (tapping mode), which gave the exact view of the surface area. The peaks below the zero point shows that there are large spaces between the two atomic structures.

\subsubsection{In vivo degradation and biocompatibility}

The figures 3.6a, and 3.6b give the scanning electron micrographs of in vivo degradation of FC film on $15^{\text {th }}$ post implantation day (PID). Individual fibers of the network are exposed due to degradation (Fig.3.6a) and the strands of the network are being covered with cells that phagocytized the material also can be seen Fig. 3.6b. The $30^{\text {th }}$ day SEM pictures show (Fig. 3.6c and 3.6d) that only remnants of the film can be seen and there is no evidence for the network structure and this indicates that the film is completely degraded by the system.

The figures 3.7, which are the histological section taken on $5^{\text {th }}$ PID reveals that the FC film is surrounded by serofibrinous inflammatory exudates containing mostly neutrophils and a few macrophages. The film appears as uniform thick layer. Eosinophilic and polymorphs were seen appearing in the edges of the 
membrane and dermis. On the $10^{\text {th }}$ PID we find mostly macrophages along with a few neutrophils invading the film and there is intense fibrovascular tissue formation around them. The $15^{\text {th }}$ PID section shows that the film is gradually removed by macrophagic action. Fibrovacular tissue is evident around the film. A few degenerated and necrotic neutrophils were seen along the border of the film. From the $30^{\text {th }}$ PID sections it can be visualized that the area is filled with fibrous tissue and collagen with scattered macrophages. Surface is covered by thin layer of epithelium. Scattered and focal mononuclear cell infiltration was evident. Cluster macrophages containing phagocytized material in the cytoplast with abundant fibroblasia and collagen deposition can also be seen.

\subsubsection{Equilibrium Water Content}

From the table 3.1 it is inferred that as the fibrin content is varied the \% EWC first increases then decreases. The highest value is exhibited by $\mathrm{F}_{10} \mathrm{C}$. The $\% \mathrm{EWC}$ of any film is directly related to its porosity and the hydrophilic moieties in the system. Fibrin is a highly fibrous protein and as it contains hydrophilic groups like - $\mathrm{OH}$, - $\mathrm{COOH}-$ and $-\mathrm{NH}_{2}$ on its backbone it acts like a hydrogel (Noorjahan et al,2004). Further the macropores formed due to its network like structure enhances the water absorption capacity. So when the fibrin content is increased its \% EWC should increase but in this case it first increases and then decreases. It implies that the porosity of the films also first increases and then decreases. The lower $\%$ EWC exhibited by $\mathrm{F}_{15} \mathrm{C}, \mathrm{F}_{20} \mathrm{C}$ and $\mathrm{F}_{25} \mathrm{C}$ films may be attributed to the increased thickness of the film with the increase in fibrin content and thereby preventing the complete removal of porogen (PVP) during heat treatment which in turn has reduced the porosity of the films. The ratio of fibrin: chitosan and also the thickness of the film have favoured $\mathrm{F}_{10} \mathrm{C}$ in attaining maximum porosity and hence highest $\%$ EWC when compared to other ratios.

An ideal dressing would control the evaporative water loss from a wound, at an optimal rate. The water vapor transmission rate for normal skin is $204 \mathrm{~g} / \mathrm{m}^{2}$ per day, while that for injured skin can range from $279 \mathrm{~g} / \mathrm{m}^{2}$ to $5138 \mathrm{~g} / \mathrm{m}^{2}$ per day depending on the nature of the wound. The water vapor permeability of a wound dressing should prevent both excessive dehydration as well as build up of exudate. It was recommended that a rate of $2500 \mathrm{~g} / \mathrm{m}^{2}$ per day would provide an adequate level of moisture without risking wound dehydration. The thickness of the FC film increases with the addition of fibrin, since the amount of chitosan and PVP are kept constant. For the films $\mathrm{F}_{15} \mathrm{C}, \mathrm{F}_{20} \mathrm{C}$ and $\mathrm{F}_{25} \mathrm{C}$ the leaching out of PVP is not that effective due to the increase in the thickness of the films. Even though the thickness of $\mathrm{F}_{5} \mathrm{C}$ was lesser than that of the $\mathrm{F}_{10} \mathrm{C}$ it exhibited lower WVTR value. This may be due to the lower fibrin content and hence lower porosity. Hence $\mathrm{F}_{10} \mathrm{C}$ would prove a better dressing as far as WVTR is concerned.

The network structure in $\mathrm{F}_{10} \mathrm{C}$ xerogel (Fig.3.2a) and hydrogel (Fig 3.2b) is retained and this indicates that the film is porous and has space to retain water particles within the system. From the SEM photos of xerogel it is clear that a mutual compatibility exists between fibrin and chitosan. The strands of fibrin expanding throughout the surface could also be visualized. Micropores formed by leaching the porogen PVP ( molecular size) could not be visualized through SEM and this can be explained as chitosan and PVP are miscible in molecule level and extremely strong hydrogen bonding interaction exists between them (Zeng et al 2004).

The emergence of large grooves in Figs 3.2 a \& b (arrow marks) and extensive rough morphology in degraded samples indicate the progress of degradation in the hydrogels. The remnants of the film on $30^{\text {th }}$ day reveals no evidence of network structure and this indicates that the film is completely degraded by the system.

The tissue reactions observed in the rat subcutaneous model suggest a comparatively reasonable compatibility in the physiological environment. The FC film elicited slight inflammatory responses, but there was a total absence of tissue necrosis with no evidence of any extended chronic inflammation. It is well acknowledged that the inflammation around the implant is a process of the normal host defense mechanism brought about as a result of surgical implantation (Goglewski et al 1993, Ibim et al 1998). The initial inflammatory response subsided gradually by day 10 with intense fibrovacular tissue proliferation. The adhered macrophages can produce reactive oxygen intermediates (ions and free radicals) in response to certain stimuli (Kao et al 1994) and these species subsequently initiate degradation at the implant-cell interface (Zhao et al 1992). Similarly, degradation and pitting of the film is attributed to release of reactive oxygen intermediate at the FC-cell interface. Platelet aggregation on fibrin surfaces has been reported (Skarja et al 1998) and degradation of fibrin proceeds by the action of plasmin (Bence et al 1999). Complete degradation of FC film was observed on 30 PID and hence it is concluded that it is biocompatible, biodegradable and can serve as a potential wound dressing material.

\subsection{Conclusions}

The FC films prepared using fibrin $10 \mathrm{ml}(40 \%$ solids) and $5 \mathrm{ml}$ of $4 \%$ chitosan solution in $0.3 \mathrm{~N}$ acetic acid have shown better percentage equilibrium water content (\% EWC), water vapor transmission rate (WVTR) and antibacterial penetration. This membrane was also implanted subcutaneously in the rats for its biodegradability. The scanning electron micrographs have shown the biodegradation of FC film by 30 days. The material has not shown any acute inflammatory or foreign body reaction at the implant site indicating that the material is biocompatible. 


\section{REFERENCES}

[1]. Alain D, D. Monique, Chitosan: structure-properties relationship and biomedical applications, in: S. Dumiprin (Ed.), Polymeric Biomaterials, second ed., Marcel Dekker, New York, 2002, 187-212.

[2]. Anderson JM. In vivo biocompatibility of implantable delivery systems and biomaterials. Eur J Pharm Biopharm 1994; 40: 1-8.

[3]. Bence CA, Woodhouse KA. Plasmin degradation of fibrin coatings on synthetic polymer substates. J Biomed Mater Res; 46: 1999, 305-314.

[4]. Berthod F, Hayek D, Damour O, Collombel C., Collagen synthesis by fibroblast cultured within a collagen sponge. Biomaterials; 14: 1993749-54.

[5]. Biagini G, Bertani A, Muzzarelli R, Damadei A, DiBenedetto G, Belligolli A, Riccotti G, Zucchini C, Rizzoli C., Wound management with N-carboxybutyl chitosan. Biomaterials; 12: 1991 281-5.

[6]. Bulter B.L., P.J. Vergano, R.F. TEstin, J.M. Bunn and J.L. Wiles, Mechanical and barrier properties of edible chitosan films as affected by composition and storage. J Food Sci 61 (1996), pp. 953-961.

[7]. Boateng JS, Matthews KH, Stevens HN, Eccleston GM, Wound healing dressings and drug delivery systems: a review,. J Pharm Sci. Aug;97(8): 2008,2892-923.

[8]. Changez M, Koul V, Krishna B, Dinda AK, Choudhary V.,Studies on biodegradation and release of gentamicin Biomaterials. ;25(1),2004, 139-46.

[9]. Chun-mao Han, Li-ping Zhang, Jin-zhang Sun, Hai-fei Shi, Jie Zhou and Chang-you Gao, Application of collagenchitosan/fibrin glue asymmetric scaffolds in skin tissue engineering, Journal of Zhejang University-Science B (Biomedicine and Biotechnology), ISSN:1673-1581

[10]. Corkhill PH, Jolly AM, Ng CO, Tighe B, J. Polymer. 28: 1987,1758.

[11]. Cuadrillero Martin F, Sandoval Cuadrado, Rodriquez Hernandez, Hydro colloidal dressings. Their effectiveness in radio dermatitis Rev Enferm.; 27(5): 2004,17-22

[12]. Değim Z., Use of microparticulate systems to accelerate skin wound healing. J Drug Target, July, 16(6): 2008, $437-$ 48.

[13]. Cuadrillero Martin F, Sandoval Cuadrado P, Rodriguez Hernandez F. Collagen powder: Hydrocolloidal dressings. Their effectiveness in radiodermatitis. Rev Enferm 2004;27:17-22.

[14]. Gennadios, A.; Weller, C.L. Gooding, C.H. Measurment errors in vapor permeability of highly permeable, hydrophilic edible films J.Food Eng.; 21: 1994, 395

[15]. Georgiev I.L., I.G. Illiev, M.K. Asenova and F. Kiril, Treatment of waste water from distilleries with chitosan. Water Res 34 2000, 1503-1506.

[16]. Ghayamzadeh R, Haddadi-Asl V, Mirzadeh H., Bioadhesion and biocompatibility evaluations of gelatin and polyacrylic acid as a crosslinked hydrogel in vitro. J Biomater Sci Polym Ed.;15(8): 2004,1019-31.

[17]. Gogolewski, S., Jovanovic, M., Perren, S.M., Dillion, J.G., Hughes, M.K., Tissue response and in vivo degradation of selected polyhydroxyacids:polylactides (PLA), poly(3-hydroxy butyrate) (PHB) andpoly(3-hydroxybutyrate-co3 hydroxyvalerate) (PHA/VA). J. Biomed. Mater. Res. 27, 1993,1135-1148.

[18]. Gong H.P., Y.H. Zhong, J.C. Li, Y.D. Gong, N.M. Zhao and X.F. Zhang, Studies on nerve cell affinity of chitosanderived materials. J Biomed Mater Res, 52 2000,. 285-295.

[19]. Hahn SK, Jelacic S, Maier RV, Stayton PS, Hoffman ASAnti-inflammatory drug delivery from hyaluronic acid hydrogels.J Biomater SciPolymEd.;15(9), 2004, 1111-9.

[20]. Haiguang Zhao, Lie Ma, Jie Zhou, Zhengwei Mao, Changyou Gao and Jiacong Shen, Fabrication and physical and biological properties of fibrin gel derived from human plasma,Biomed.Mater. Volume 3, Number 1, 2008,281289.

[21]. Hunt JA, Vince DG, Williams DF. Image analysis in the evaluation of biomaterials. J Biomed Engineering, 15: 1993; 39-45.

[22]. Ibim, S.M., Uhrich, K.E., Bronson, R., Amin, S.F., Langer, R.S.,Laurencin CT. Poly(anhydride-co-imide): in vivo biocompatibility in a rat model, Biomaterials; 19, 1998,941-951.

[23]. Jayasree RS, Rathinam K, Sharma CP. Development of artificial skin (Template) and influence of different types of sterilization procedures on wound healing pattern in rabbits and guinea pigs. J Biomater Appl; 10: 1995, 144162.

[24]. Jiali Zhang, Wenshui Xia, Ping Liu, Qinyuan Cheng, Talba Tahirou, Wenxiu , Chitosan Modification and Pharmaceutical/Biomedical Applications, Mar. Drugs, 8(7), 2010,1962-1987.

[25]. Kao W.J, Hiltner A., J.M. Anderson, G.A. Lodoen, Theoretical analysis of in vivo macrophage adhesion and foreign body giant cell formation on strained poly(etherurethane urea) elastomers, J. Biomed. Mater. Res. 28 , 1994, 819-829.

[26]. Kwunchit.O and W.M. Bernd, Chitosan sponges as sustained release drug carriers. Int J Pharm 156, 1997, 229237.

[27]. Majno, G. The Healing Hand: Man and Wound in the Ancient World, Harvard University Press, Cambridge, 1975.

[28]. Martin P. Wound healing - aiming for perfect regeneration Science, 1997; 276:75-81

[29]. Mi FL, Shyu SS, Wu YB, Lee ST, Shyong JY, Huang RN. Fabrication and characterization of a sponge-like asymmetric chitosan membrane as a wound dressing. Biomaterials; 22, 2001, 165-173.

[30]. Minami S, Okamoto Y, Tanioka S, Sashiwa H, Saimoto H, Matsuhashi A, Shigemasa Y. Effects of chitosan on wound healing; in Carbohydrates and carbohydrate polymers (ed) M Yalpani (Illinois: ATL Press) 1992,141-152.

Mochizuki A, Sato Y, Ogawara H, Yamashita S. Pervaporation separation of water/ethanol mixtures through polysaccharide membranes, The effects of salts on the permselectivity of cellulose membrane in pervaporation. $\mathbf{J}$ Appl Polym Sci; 37,1989, 3357 - 3374. 
[31]. Muzzarelli R, Tarsi R, Filippini O, Giovanetti E, Biagini G, Varaldo PE. Antimicrobial properties of Ncarboxybutyl chitosan. Antimicrob Agents Chemother; 34, 1990, 2019-23.

[32]. Muzzarelli RA, Isolati A, Ferrero A. Chitosan membranes, ion exchange and membranes. Vol. 1. London: Gordon and Breach, 1974, 193-6.

[33]. N. Natarajan, V. Shashirekha, S. E. Noorjahan, M. Rameshkumar C. Rose, T. P. Sastry, Fibrin-Chitosan-Gelatin Composite Film: Preparation and Characterization, Journal of Macromolecular Science, Part A, Volume 42, Issue 7 July 2005 , pages $945-953$.

[34]. Naomi Eidelman, Combinatorial screening of cell proliferation on poly blends, Journal of biomaterial, Vol.26 (34), (2004), 6906-6915.

[35]. Noorjahan SE, Sastry TP. An in vivo study of hydrogels based on physiologically clotted fibrin-gelatin composites as wound-dressing materials. J Biomed Mater Res Part B: Appl Biomater; 71B: 2004,305-312.

[36]. Noorjahan SE, Sastry TP. Hydrogels based on physiologically clotted fibrin-gelatin composites. J Polym Sci Part A: Polym Chem; 42,2004, 2241-2252.

[37]. Ong SY, Wu J, Moochhala SM, Tan MH, Lu J., Development of a chitosan-based wound dressing with improved hemostatic and antimicrobial properties. Biomaterials. 2008 Nov;29(32),2008,4323-32.

[38]. Ravindra Babu P, Sastry TP, Rose C, Muralidhara Rao N. Hydrogels based on gelatin poly(hydroxyethyl methacrylate) and poly(butylacrylate) graft copolymer impregnated with fibrin. J Appl Polym Sci; 65, 1997, 555560.

[39]. Rothwell SW, Fudge JM, Reid TJ, Krishnamurti C. Epsilon-amino caproic acid additive decreases fibrin bandage performance in a swine arterial bleeding model.Thromb Res. Dec 15;108(5-6), 2002, 341-5.

[40]. Rubens BD, Perry DW, Hatton MWC, Bishop PD, Packham MA, Kinlough- Rathbone RL. Thromb Haemostas.; 73, 1995, 850.

[41]. Sathian J, Sastry TP, Suguna L, Lakshminarayana Y, Radhakrishnan G. Fibrin as a matrix for grafting 2hydroxyethyl methacrylate: Preparation and characterization of the graft and its in vivo evaluation for wound healing. J Biomed Mater Res;65A,2003, 435-440.

[42]. Schalag G, Redl H, 1986, Eds. Fibrin sealant in generative medicine, vol.1-5, Springer Berlin Heidelberg, New York.

[43]. Segura T, Chung PH, Shea LD. DNA delivery from hyaluronic acid-collagen hydrogels via a substrate-mediated approach.Biomaterials.; 26(13), 2005,1575-84.

[44]. Skarja GA, Brash JL, Bishop P, Woodhouse KA. Protein and platelet interactions with thermally denatured fibrinogen and cross-linked fibrin coated surfaces. Biomaterials; 19:2129-2138.

[45]. Sripriya R, Kumar MS, Sehgal PK.2004, Improved collagen bilayer dressing for the controlled release of drugs. J Biomed Mater Res. ;70B(2),1998,389-96.

[46]. Su CH, Sun CS, Juan SW, Hu CH, Ke WT, Sheu MT. Fungal mycelia as the source of chitin and polysaccharides and their applications as skin substitutes. Biomaterials; 18, 1997, 1169-74.

[47]. Sun LP, Wang S, Zhang ZW, Wang XY, Zhang QQ. 2009, Biological evaluation of collagen-chitosan scaffolds for dermis tissue engineering. Biomed Mater.; 4(5), 487- 501.

[48]. Thomas S., 1990, Wound Management and Dressing. London, Pharmaceutical Press.

[49]. Tomihata K, Ikada Y. In vitro and in vivo degradation of films of chitin and its deacetylated derivatives. Biomaterials; $18,1997,567-73$.

[50]. Tokura S, S.I. Nishimura, N. Sakairi and N. Nishi, Biological activities of biodegradable polysaccharide. Macromol Symp 101,1996, 389-396.

[51]. William GM, Herbert JQ. 1985, Method of achieving hemostasis inhibiting fibroplasias, and promoting tissue regeneration in a tissue wound. United States Patent No. 4532134.

[52]. Xu, S.P. McCarthy and P.A. Gross, Chitosan film acylation and effects on biodegradability. Macromolecule 29, 199), pp. 3436-3440

[53]. Ye Q, Zund G, Benadikt P, Jockenhoevel S, Hoerstrup SP, Sakyama S, Hubell JA, Turina,, Eur J Cardio Thorasic Surg, 2000, 17(5), 587.

[54]. Ye Q, Zünd G, Benedikt P, Jockenhoevel S, Hoerstrup SP, Sakyama S, Hubbell JA, Turina M. Fibrin gel as a three dimentional matrix in cardiovascular tissue engineering Eur J Cardio Thorcic Surg.; 17(5), 2000, 587-591

[55]. Zeng M, Fang Z., Xu C. Effect of compatibility on the structure of the microporous membrane prepared by selective dissolution of chitosan/synthetic polymer blend membrane. J Membrane Sci 230, 2004,175-181.

[56]. Zhao Q. Anderson J.M, A. Hiltner, G.A. Lodoen, C.R. Poyet, Theoretical analysis of cell size distribution and kinetics of foreign body giant cell formation on strained polyurethane elastomers, J. Biomed. Mater. Res. 26, 1992, $1019-1038$

[57]. Wedmore, Ian, McManus, John G, Anthony E, A Special Report on the Chitosan-based Hemostatic Dressing: Experience in Current Combat Operations, Journal of Trauma-Injury Infection \& Critical Care: March 2006 Volume 60 - Issue 3, 2006,655-658

[58]. Paul Baldrick, The safety of chitosan as a pharmaceutical excipient, Regulatory Toxicology and Pharmacology Volume 56, Issue 3, April 2010, Pages 290-299. 